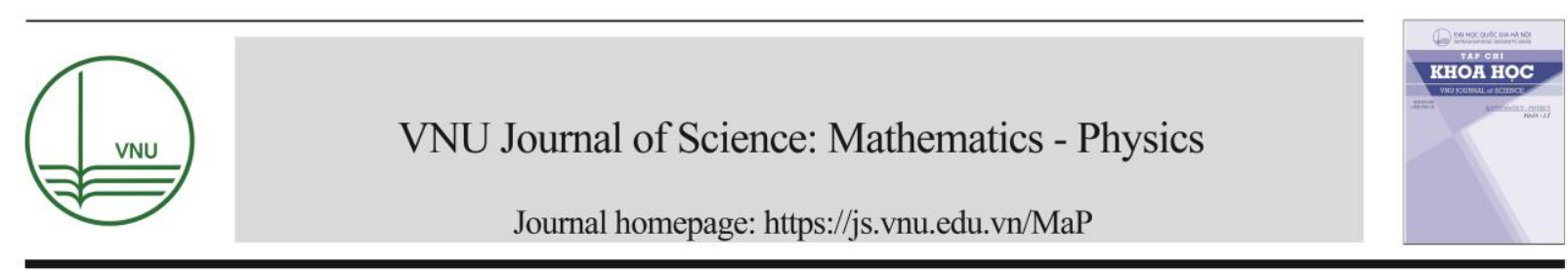

\title{
Building Low Power Wireless Sensor Network with TCP/IP for Agriculture
}

\author{
Le Quang Thao*, Dinh Thi Hai, Tran Thanh Ha \\ Faculty of Physics, VNU University of Science, 334 Nguyen Trai, Hanoi, Vietnam \\ Received 28 June 2018 \\ Revised 04 August 2018; Accepted 20 August 2018
}

\begin{abstract}
This paper presents a low power Wireless Sensor Network using TCP/IP to use Ethernet configuration and hybrid networks to extend range or improve connectivity in distant areas, in particular is Agriculture. The system is designed with soil moisture sensor based on Atmega8L to monitor environmental parameter. The Nordic nRF24L01 was used to transmit the obtained data to the Web server based on Ethernet configuration. Moreover, the SIM800L module was used to upload these data based on GSM/GPRS service in order to make data available globally. The fabricated system not only uses a low power but also has a compact size and applies advantages of TCP/IP.
\end{abstract}

Keywords: Smart wireless sensor network, Internet of Things, consumption energy.

\section{Introduction}

Wireless sensor network is the combination of hundreds or thousands of sensor nodes which help in sensing, self-computation, signal processing and communication mechanisms [1]. The design of a WSN depends significantly on the applications and has more advantages than traditional network, which keeps maximize operation lifetime and minimize the cost [2].

In this paper, we have designed and implemented of a Wireless Sensor Network (WSN) node that utilizes for agriculture with low cost components and saving energy. We have used soil moisture sensor based on Atmega8L microcontroller of Atmel which is designed to optimize the device for power consumption versus processing speed. The consumed power of WSN is evaluated by several cycle of sleep, wake up and run. The GSM/GPRS module would send the acquired data directly to the Internet using TCP/IP protocol. This WSN system could store unlimited data in Web server that is a good candidate for application of agriculture.

\footnotetext{
*Corresponding author. Tel.: 84-983712941.

Email: thaolq@hus.edu.vn

https//doi.org/ 10.25073/2588-1124/vnumap.4275
} 


\section{Materials and methods}

The fabricated system consists of four sensor nodes and the central. The main function of sensor node is monitoring environment parameter, performing local data processing, and broadcasting the data to the central. The central receives processed data, sends them to Internet and then stored in Web server.

\subsection{The sensor node}

Block diagram and components of a sensors node is shown in Figure 1 that is indispensable hardware components which are connected with Atmega8L [3], which is high-performance, low power Atmel AVR 8-bit microcontroller. The sensor node's, energy is consumed by the sensor, the microcontroller unit and the transceiver modules. They were maintained by a limited power supplies such as battery.

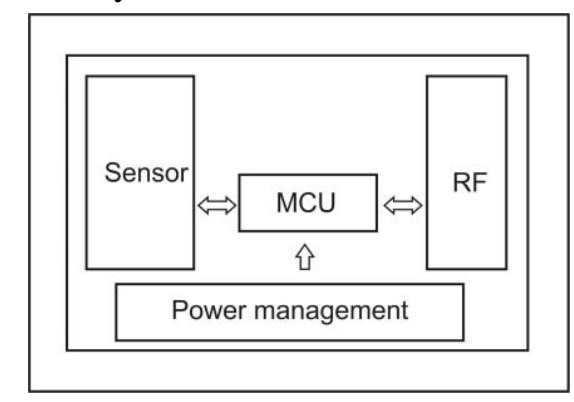

Figure 1a. Block diagram of sensor node.

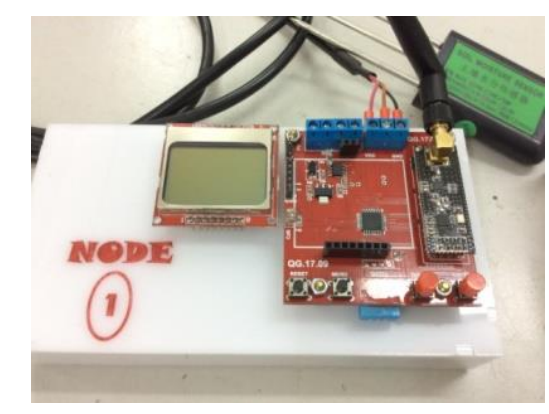

Figure 1b. Components of a sensor node.

In this sensor node, the nRF24L01 module was used as the transceiver, which is produced by Nordic Semiconductor [4] and enhanced with a ShockBurst hardware protocol accelerator and high speed interface with extremely low power requirement. The MS10 soil moisture sensor [5] was used to measure the dielectric constant of the reaction of soil, soil direct stable real moisture content using with high accurate and high sensitive. This sensor is convenient for precision Agriculture to monitor parameter accurately.

Sensor nodes have three modes of operation: sleep, wake up and active. Almost of time, nodes are sleep mode, in this case the microcontroller still maintains its state and consumes a little power while nRF24L01 module shuts down or disconnects. After acquiring, the obtained data will be processed by the microcontroller and compared with the threshold. If the data exceeds the threshold, it will be sent to the central to store in the Web server and control water pump.

\subsection{The central}

The diagram of central consists of transceiver module, interface block, real-time module, SIM800L module and the power controller. All of these blocks will be controlled by microcontroller and the block diagram and components are shown in Figure 2a and Figure $2 b$.

To send and receive data based on GPRS service over TCP/IP protocol, we use the SIM800L module. This module is a complete Quad-band 850/900/1800/1900MHz GSM/GPRS embedded AT command interface. It is allowed total cost savings and fast time-to-market for customer applications [6]. When the data are received, they will be sent to the internet with activated GPRS functionally and provide a TCP/IP based connection toward server. 


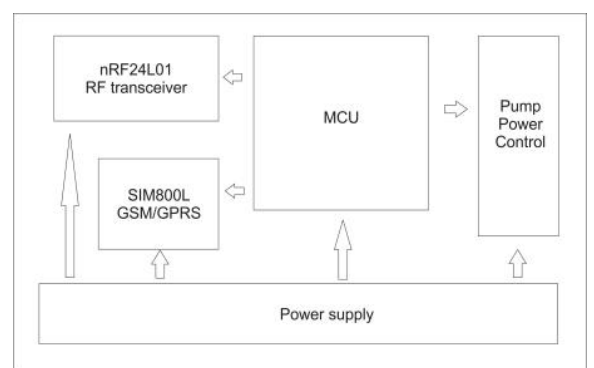

Figure 2a. Block diagram of the central

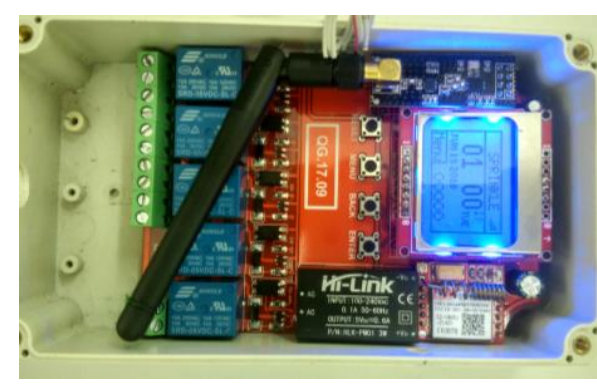

Figure 2b. Components of the central

\section{Results and discussion}

In this section, we examine the energy consumption in sensor nodes and the operation to send data directly to internet by GPRS service in the central.

\subsection{Sensor node current consumption measurements}

In WSN, the most of energy is consumed by transmission and reception processes [7]. In this study, the current consumption of the fabricated WSN has evaluated during the sleep, active modes and the transmission process. As usually, data will be transmitted right after being received, this work causes lost energy because maybe data is not useful. The advantage of our approach is data woull be processed and compared before transmitting. This approach can save more energy than others. In the experiment, we used Shunt resistance [8] method with a low-impedance selected to minimize the voltage drop in the supply line of the node. Therefore, the current consumed by node was measured directly by the oscilloscope DL1720E as shown in the block diagram in Figure 3.

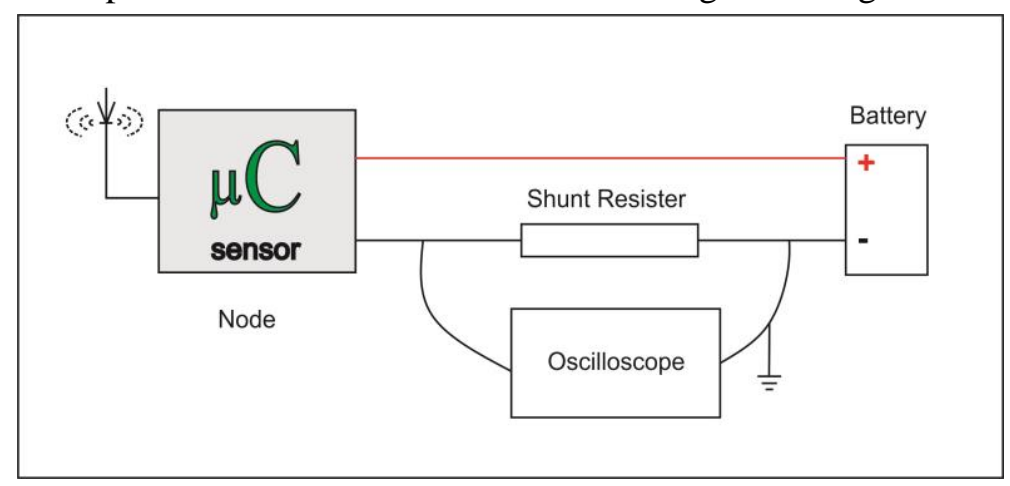

Figure 3. Configuration measurements of current consumption.

The voltage across the Shunt resistance is proportional to the current flowing through it so that the power consumption of the sensor node is calculated as following:

$$
\mathrm{P}_{\text {Node }}=\mathrm{V}_{\text {Node }} * \mathrm{I}=\left(\mathrm{V}_{\text {Battery }}-\mathrm{V}_{\text {Shunt }}\right) * \mathrm{~V}_{\text {Shunt }} / \mathrm{R}_{\text {Shunt }}
$$

where: $\mathbf{P}_{\text {Node }}$ is the total energy in node; $\mathbf{V}_{\text {Node }}$ is the voltage that shown in the oscilloscope; $\mathbf{I}$ is the current; $\mathbf{V}_{\text {Battery }}$ is the voltage on battery; $\mathbf{V}_{\text {Shunt }}$ is the voltage on Shunt Resistor and $\mathbf{R}_{\text {Shunt }}$ is $8.3333 \Omega$. 
To measure current consumption, we built a program let sensor node sleep 1 second and then read data from soil moisture sensor and send data without acknowledgement signal and this process is repeated continuously. This measurement was shown in Figure 4.

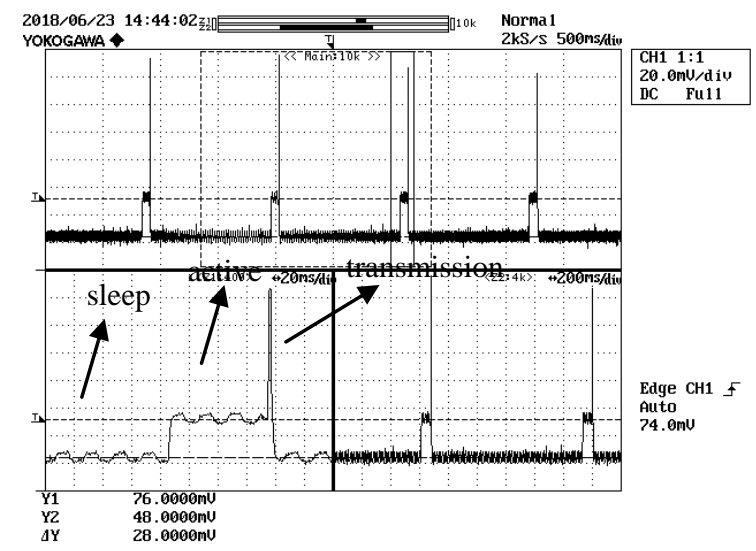

Figure 4a. Current consumption during sleep and active mode.

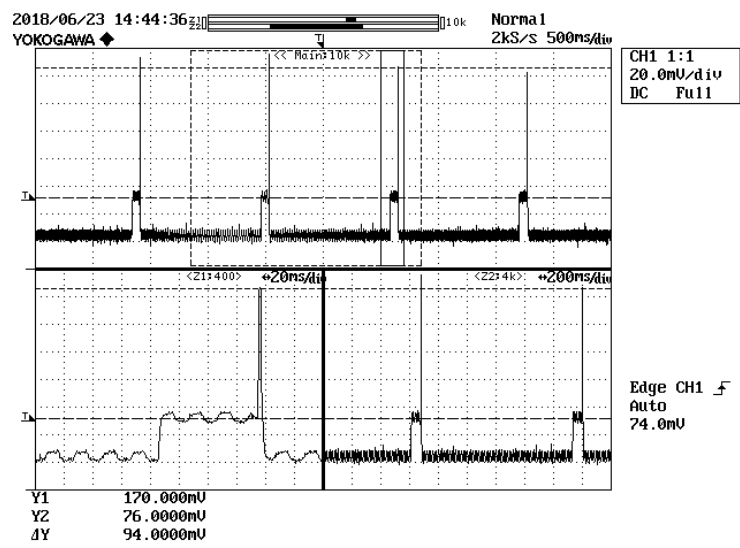

Figure 4b. Current consumption during transmission.

According to Figure 4, the current consumption in sleep and active modes were $48 \mathrm{mV} / 8.333 \Omega=$ $5.76 \mathrm{~mA}$, and $(48+28) \mathrm{mV} / 8.333 \Omega=9.12 \mathrm{~mA}$ respectively, and one of the transmition was $(94+28+48) \mathrm{mV} / 8.333 \Omega=20.40 \mathrm{~mA}$. These currents indicate that even nodes is in sleeping it still wastes energy. To advoid energy waste, in the transmition mode, obtained data are only sent when they are exceeded the threshold value and this is the most advantage of our approach.

\subsection{Sending data to Web server using TCP/IP}

Data will be processed and sent following the steps such as bring up wireless connection with GPRS, configuring Domain Name System, connecting and sending data with the AT commands which are provided by SIMCOM. The following website has been developed to store and display environment data on internet using SQL server and PHP platform. The front end and database of website are shown in Figure 5a and Figure 5b.

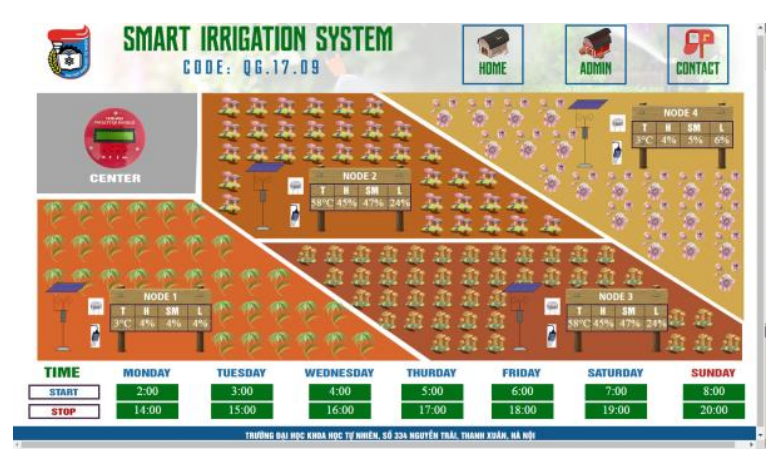

Figure 5a. Front-end Website interface.

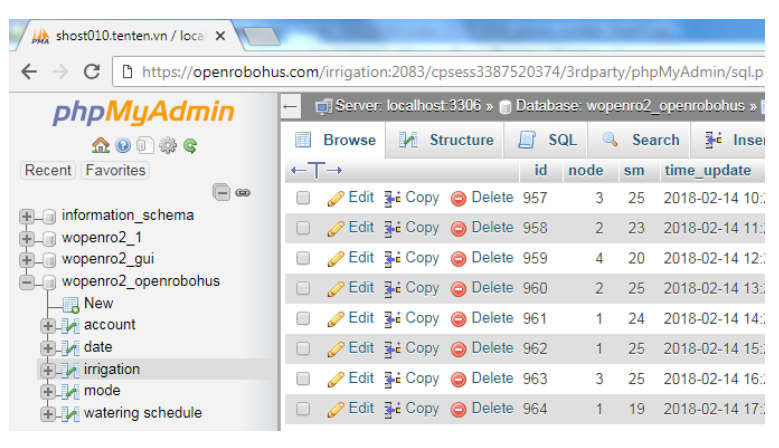

Figure 5b. Data on Mysql server. 


\section{Conclusion}

In this article, the WSN system including the central and 4 sensor nodes was successfully designed and fabricated. The consumption current of sensor node was measured to be $5.76,9.12$, and $20.40 \mathrm{~mA}$ corresponding to sleep, active, and transmition modes, respectively. Especially, the measured data of environmental parameters were managed in real time. These data were also sent to SQL server and PHP platform based on GRPS service or WIFI using TCP/IP protocol that can monitor and control environment easily by using IoT technology. To save consumption energy of system, the obtained data just send when exceeding threshold. It is indicated that system brings the convenient of TCP/IP in sensor node which makes more delightful applications for WSN to Agriculture in future.

\section{Acknowledgments}

This work is implemented with the help of the Project QG.17.09 that is supported by the VNU University of Science. I would like to thank for the help

\section{References}

[1] I. Akyildiz, W. Su, Y. Sankarasubramaniam and E. Cayirci. A survey on Sensor Networks. IEEE Communications Magazine, Vol. 40, Issue: 8, pp. 102-114, August 2002.

[2] V. Kumar, S.S. Sonavane, B.P. Patil, Designing Ultra Low Power Wireless Sensor Network with TCP/IP link, DOI: 10.1109/ICASTECH.2009.5409742.

[3] https://www.microchip.com/wwwproducts/en/ATmega8 accessed on 28th May, 2018

[4] Nordic Semiconductor Corp, nRF24L01 Ultra low power 2.4GHz RF Transceiver, Technical specification.

[5] MS10 soil moisture and temperature sensor User's manual. http://www.szbonad.com/MS10 accessed on 10th June, 2018

[6] Sim800L technical specification, http://simcom.ee/documents/?dir=SIM800 accessed on 17th, June, 2018

[7] Alippi, C.; Anastasi, G.; Di Francesco, M.; Roveri, M. Energy management in wireless sensor networks with energy-hungry sensors. IEEE Instrum. Meas. Mag. Vol. 12, N. 2, April 2009, pp. 16-23.

[8] https://www.rc-electronics-usa.com/current-shunt.html accessed on $16^{\text {th }} \mathrm{June}, 2018$. 